\title{
ELLIPTICAL GATE MOSFETS A MODELING APPROACH
}

\author{
Anu Puri ${ }^{1}$, Parul Wadhwa ${ }^{2}$ \\ ${ }^{1,2}$ Assistant Professor, NHCE, Bangalore \\ anu.puri2015@gmail.com ${ }^{1}$,parulwadhwa5@gmail.com ${ }^{2}$
}

\begin{abstract}
The demand for higher current drives, better short channel characteristics has led to the evolution of multiple gate mosfets from classical single gate devices. Silicon gate all around mosfets(GAA) offers maximum gate control over the channel which makes them an excellent choice among non classical MOS devises. However GAA mosfets with circular cross sections are not common in real fabrication. Elliptical Cross sections have been reported. In this paper new modeling approach for elliptical GAA have been investigated, dependence of physical properties of a device on the behavior of multiple gate devices have been discussed.
\end{abstract}

Keywords: Circular, Effective Radius, SCE (Short Channel Effects), Elliptical, Gate-All-Around (GAA), Nanowire, Aspect Ratio

\section{INTRODUCTION}

Scaling of MOS Devices is very important in order to achieve higher packing density \& better performance VLSI circuits. As we move down in channel length, short channel effects arise like threshold voltage roll-off, drain induced barrier lowering (DIBL), hot carrier effect etc.. Gate controllability reduces as we move down in channel length. Hence threshold voltage starts depending on channel length and other problems like poor subthreshold slope, less transconductance, high drain conductance, poor Ion/Ioff ratio that is less on current $\&$ high off current, hot carrier effect etc arise

\section{Multiple Gate Mosfets(In search of perfect Switch)}

The problems associated with scaling are huge and classical mosfets are no longer behaving as a perfect switch. Multiple Gate Mosfets also known as non classical devices getting there name from multi (more than one gate) such as double, triple, quadruple,pi gate etc. have shown better performance as the total control of the gate over the channel region increases. They help improve gate controlability and also reduce SCE. These devises help improve efficiency of mosfets in the following areas.

- Lower VT roll-off as the gate has better control over the channel so even when we reduce length, threshold voltage does not change much

- Lower DIBL (Drain induced barrier lowering) as the Multiple gate mosfets help prevent electric field lines from the drain to encroach the channel region.

- Better subthreshold slope

- Better Ion/Ioff ratio which means they can behave almost like a perfect switch with high on current \& very less off current Better transconductance and drain conductance

- Natural Length: Multiple gate Mosfets also have shown improved valves of Natural length which is defined as the length of the channel region controlled by the drain.
A device is free of short channel effects if the effective gate length of a MOS device is larger than 5 to 10 times the natural length.This natural length can be reduced by decreasing gate oxide thickness, decreasing silicon film thickness, by using high $\mathrm{K}$ gate dielectric instead of silicondioxide.

Natural length can also be reduced when the number of gates are increased. Since in multiple gate Mosfets we are increasing the number of gates, so we will get a lower natural length

However efficiency of multiple gate devices devices depend on certain physical dimensions

Dependence on device physical Dimensions

- Doping Concentration

- $\quad$ Silicon film thickness

- Channel width

- $\quad$ Oxide thickness

DOPING CONCENTRATION: Increase of doping concentration is supposed to decrease short channel effects like DIBL, Vt roll-off as it helps decrease penetration of the drain electric field in to the channel. However it leads to the threshold voltage fluctuation.

SILICON FILM THICKNESS: Even though the use of thin silicon film thickness increases source and drain resistance, a small silicon film thickness is required to reduce short channel effects.

However for double gate Mosfets, it also depends on what is the gate length being used.

The threshold voltage of double gate devices with $\mathrm{L}=30 \mathrm{~nm}$ decreases when the film thickness is increased but it increases with film thickness if gate length is larger than 40nm.[6] 
CHANNEL WIDTH: DIBL, Subthreshold slope increases with increase of channel width as it becomes easier for the electric field from drain to encroach in the channel region which also increases the threshold voltage.

GATE OXIDE THICKNESS The gate oxide thickness if reduced will lead to gate tunneling current and would effect the output drain current. Hence thickness of gate oxide has to be in prescribed limits which does not effect the device parformance

\section{ELLIPTICAL GATE MOSFETS}

As discussed it is becoming very important that we move from classical to nonclassical MOS devises which provide excellent gate coverage and better improved characteristics. Among multiple gate devises GAA (gate all around) mosfets have shown best results. So it is indeed the structure for next generation nano devises. Due to manufacturing limitation GAA devises are not able to take their theoretically round shape. Elliptical shaped surrounding mosfets have been reported [1][2]. Hence several studies on elliptical GAA were performed.

\section{ELLIPTICAL GATE MOSFETS (NEW MODELING APPROCH)}

Bangsaruntip et al [1] proposed the universality of SCE In undoped elliptical GAA mosfets. Li and HWANG [3] studied the aspect ratio of GAA mosfets by numerical simulation. ZHANG et al [4] proposed the concept of effective radius to convert elliptical GAA mosfets in to equivalent circular GAA mosfets for studying short channel effects. They found that the elliptical mosfets that have the same effective radius exhibit the same short channel effect. The aspect ratio (AR) is defined by the ratio of the length of major axis to minor axis of an ellipse shaped surrounding gate MOS devises. TCAD simulation results have shown the sensitivity of drain-induced barrier lowering (DIBL)and other short channel effects to the variation in aspect ratio. Hence elliptical GAA has significant impact on SCE in mosfets. Aspect ratio if one give perfect circular cross section

Zhang et al proposed that for an undoped or lightly doped GAA nano wire in the subthreshold region mobile charge is negligible, hence 3D Poisson equation reduces to laplace equation

$d^{2} \phi / d x^{2}+d^{2} \phi / d y^{2}+d^{2} \phi / d z^{2}=0$

$0 \leq \mathrm{z} \leq 1$

$\mathrm{z}$ is the axis along the channel direction.

The electrostatic potential along the radius is assumed to be parabolic

$\Phi(\mathrm{r}, \mathrm{z})=\mathrm{c}_{1}(\mathrm{z})+\mathrm{c}_{2}(\mathrm{z}) \mathrm{r}+\mathrm{c}_{3}(\mathrm{z}) \mathrm{r}^{2}$ (for cylindrical)

Where $\mathrm{r}$ is the radius of cylindrical mosfets

\section{FOR ELLIPTICAL STRUCTURES}

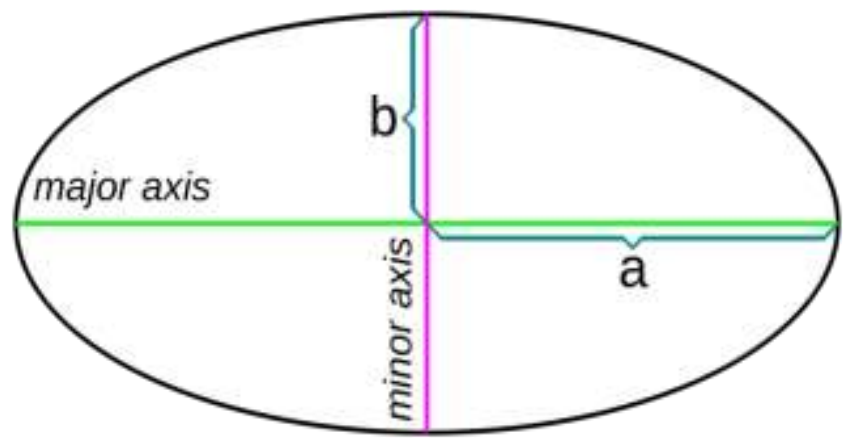

ALONG X- DIRECTION

$$
\begin{aligned}
& \Phi(\mathrm{x}, \mathrm{z})=\mathrm{c}_{1}(\mathrm{z})+\mathrm{c}_{2}(\mathrm{z}) \mathrm{x}+\mathrm{c}_{3}(\mathrm{z}) \mathrm{x}^{2}(\mathbf{1}) \\
& \text { At } \mathrm{x}=0 \text { and } \mathrm{z}=0 \\
& \Phi(\mathrm{x}, \mathrm{z})=\Phi(0, \mathrm{z}) \text { ( center potential) }
\end{aligned}
$$

Hence $\Phi(0, \mathrm{z})=\mathrm{c}_{1}(\mathrm{z})$

Differentiating equation( 1) w.r.t z

$\mathrm{d} \phi(\mathrm{x}, \mathrm{z}) / \mathrm{dz}=\mathrm{c}_{2}(\mathrm{z})+2 \mathrm{x} \mathrm{c}(\mathrm{z})$

at $\mathrm{x}=0$ and $\mathrm{z}=0$

$\mathrm{d} \phi(\mathrm{x}, \mathrm{z}) / \mathrm{dz}=0$

hence $c_{2}(z)=0$

finally we get $\phi(x, z)=\phi(0, z)+c_{3}(z) x^{2}$

\section{ALONG Y- DIRECTION}

$\phi(\mathrm{y}, \mathrm{z})=\phi(0, \mathrm{z})+\mathrm{D}_{3}(\mathrm{z}) \mathrm{y}^{2}$

Differentiating equation 2 w.r.t $\mathrm{x}$ and substitute $\mathrm{x}=\mathrm{a} \cos \omega$ $\mathrm{d} \phi(\mathrm{x}, \mathrm{z}) / \mathrm{dx}=2 \mathrm{x} \mathrm{c}_{3}(\mathrm{z})=2 \mathrm{a} \cos \phi \mathrm{c}_{\mathrm{z}}\left[\mathrm{c}_{3}(\mathrm{z})=\mathrm{c}_{2}\right]$

Applying boundary condition at $\mathrm{x}=\mathrm{a} \cos \omega$ (point on ellipse)

We get $d \phi(x, z) / d x=C_{o x} / €_{\mathrm{si}}\left(V_{\mathrm{GS}}-\phi_{\mathrm{s}}(\mathrm{z})-\mathrm{V}_{\mathrm{FB}}\right)$

Hence $c_{\mathrm{z}}=\mathrm{C}_{\mathrm{ox}} / 2 \mathrm{a} \cos \omega €_{\mathrm{si}}\left(\mathrm{V}_{\mathrm{GS}}-\phi_{\mathrm{s}}(\mathrm{z})-\mathrm{V}_{\mathrm{FB}}\right)$ now $C_{\mathrm{ox}}=€_{\mathrm{ox}} €_{\mathrm{si}} /\left(\right.$ tox $\left.€_{\mathrm{si}}+€_{\mathrm{ox}} \mathrm{a} \cos \varphi\right)$

Applying Boundary conditions at $\mathrm{y}=\mathrm{b} \sin \omega$ $\mathrm{d} \phi(\mathrm{y}, \mathrm{z}) / \mathrm{dy}=\mathrm{C}_{\mathrm{ox}} / €_{\mathrm{si}}\left(\mathrm{V}_{\mathrm{GS}}-\phi_{\mathrm{s}}(\mathrm{z})-\mathrm{V}_{\mathrm{FB}}\right)$

$\operatorname{Cox}=€_{\mathrm{ox}} €_{\mathrm{si}} /\left(\right.$ tox $\left.€_{\mathrm{si}}+€_{\mathrm{ox}} \mathrm{b} \sin \omega\right)$

Hence Substitute back in (2) and (3) we get $\Phi(\mathrm{x}, \mathrm{z})=\mathrm{C}_{\mathrm{ox}}\left(\mathrm{V}_{\mathrm{GS}^{-}} \Phi_{\mathrm{s}}(\mathrm{z})-\mathrm{V}_{\mathrm{FB}}\right) \mathrm{x}^{2} /(2 \operatorname{acos} \omega € \mathrm{si})+\Phi(0, \mathrm{z})$ $\Phi(\mathrm{y}, \mathrm{z})=\mathrm{C}_{\mathrm{ox}}\left(\mathrm{V}_{\mathrm{GS}^{-}}-\Phi_{\mathrm{s}}(\mathrm{z})-\mathrm{V}_{\mathrm{FB}}\right) \mathrm{y}^{2} /(2$ bsin $\omega € \mathrm{si})+\Phi(0, \mathrm{z})$ Substituting back in to the poisson's equation and solving it we can get the surface potential at every point of an ellipse this equation which will have a $\omega$ factor in it .Also threshold voltage at all points can be evaluated . 


\section{CONCLUSION}

This paper talks about how multiple gate Mosfets prove to be a better choice than classical MOS structure by providing better electrostatic control over the channel. Furthermore the role of other factors like channel width, gate oxide thickness, silicon film thickness, doping concentration etc also effect the performance of the device Since scaling has reached the end of the road map,Multiple gate Mosfets will certainly prove to be a better alternative over conventional MOS devices. Later part of the paper talks about a new modeling approach to solve the Poisson equation for a elliptical GAA mosfets which would we able to give an idea about surface potential at every point of an elliptical GAA mosfets and not just $\mathrm{a}$ and $\mathrm{b}$ by introducing $\mathrm{a}$ (o factor .

\section{REFERENCES}

[1]. S. Bangasarunitip, G.M Cohen, A. Majumdar, and $\mathrm{J}>\mathrm{W}$. Sleight, "Universality of short-channel effects in undoped-body silicon nanowire MOSFET's IEEE Electron Device Lett, Vol. 31, No. 9, Pp 903-905, Sep. 2010

[2]. Y.Li, and C-H. Hwang, "The effect of the geometry aspect ratio on the silicon ellipse-shaped surroundinggate field-effect transistors, and circuit, " Semicond. Sci. Technol. Vol 24, No 9, Pp. 1-8, Aug. 2009.

[3]. Y.Cui, Z. Zhong, D. Wang, W. Wang, and M. Lieber, "High performance silicon nanowire field effect transistors, "Nano Lett, Vol. 3, No. 2, Pp 149-152, Jan. 2003.

[4]. Lining Zhang, Lin Li, jin $\mathrm{He}$ and Mansun Chan, "Modeling Short-channel Effect of Elliptical Gate-AllAround MOSFETS by Effective Radius, "IEEE Electron Device Lett., Vol. 32, No. 9, Pp. 1188-1190, Sep. 2011.

[5]. N. Arora, "MOSFETS Model for VLSI Circuit Simulation Theory and Practice", New York: SpringerVerlag, 1993.

[6]. H.S.P Wong, D.J Frank and P.M Solomon,"device design Consideration for double gate ultra-thin SOI Mosfets at 25nm Channel length, in IEDM Tech dig, 1998 pp 407-410

[7]. R.H Yan, A Ourmazd,K.F Lee."Scaling the Mosfets: From Bulk to SOI to Bulk.'IEEE Trans on electron devices, VOL 39,no 7,pp1704,1992

[8]. J.T Park,J P Colinge and C.H Diaz ,'Pi gate SOI Mosfets ," IEEE electron Devices let, vol 22,pp 405406 aug 2001

[9]. C.P Auth and J.D Plummer,"A simple Model for the threshold voltage of surrounding gate mosfets, "IEEE Trans on electron devices, Vol 45,pp 2381-2383

[10].J.T Park ,C.A Collinge and J.P Collinge ," comparison of gate structures for short channel SOI Mosfets,"in proced IEEE Int SOI Conf,2001 pp 115-116 